\title{
Importância clínica do músculo esternal e suas variações anatômicas: uma revisão sistemática da literatura
}

\author{
Clinical importance of sternalis muscle and anatomical variations: \\ a systematic review of literature
}

\author{
Luana Agapito de Oliveira¹, Hananiah Tardivo Quintana², Flavia de Oliveira ${ }^{3}$ \\ ${ }^{1}$ Curso de Fisioterapia, Universidade Federal de São Paulo (UNIFESP) - Santos (SP), Brasil. \\ ${ }^{2}$ Programa de Pós-Graduação Interdisciplinar em Ciências da Saúde, UNIFESP, Campus Baixada Santista - Santos (SP), Brasil. \\ ${ }^{3}$ Departamento de Biociências, UNIFES, Campus Baixada Santista - Santos (SP), Brasil.
}

\section{RESUMO}

A presença do músculo esternal é uma variação anatômica que está situada anteriormente ao osso esterno e suas estruturas adjacentes e não tem função totalmente definida. Há na literatura mais relatos de estudos anatômicos sobre o músculo esternal do que sobre as implicações clínicas da sua presença. O objetivo deste estudo foi analisar as descrições existentes das variações anatômicas do músculo esternal, compreendendo, assim, a importância clínica desse conhecimento. Foram avaliados artigos nas línguas portuguesa e inglesa, indexados nas bases de dados PubMed e SciELO, publicados entre 1867 e 2016. Buscou-se verificar os tipos de variações anatômicas do músculo esternal descritas, suas origens e inserções, inervação e irrigação sanguínea. Em seguida, foi relatada a importância clínica do conhecimento dessa variação. O músculo esternal apresenta diversas dimensões e inserções, o que dificulta a determinação de sua função. Devido à localização paraesternal, em exames de imagem o referido músculo pode ser visto como uma nodulação anormal na região da mama e ser confundido com um tumor. O reconhecimento dessa variação antes de procedimentos cirúrgicos na parede torácica evita possíveis complicações e possibilita o uso do tecido como retalho muscular em cirurgias reconstrutivas da mama; também pode ser usado na melhora de resultados estéticos em mamoplastia de aumento ou fornecer cobertura extra para próteses. O conhecimento sobre essa variação anatômica é bastante relevante, pois a percepção da existência do músculo esternal e de suas variações relaciona-se a importantes implicações clínicas em cirurgias de mama e tórax.

Palavras-chave: esterno; variação anatômica; cirurgia torácica.

\section{ABSTRACT}

The presence of sternal muscle is an anatomical variation, located above the sternum and adjacent structures, and its function is not fully defined. In the literature, there are more reports of anatomical studies of the sternal muscle than clinical implications of its presence. The objective of this study was to analyze descriptions about anatomical variations of the sternal muscle and understand the clinical importance of this knowledge. Articles in Portuguese and English, indexed in the databases PubMed and SciELO, from 1867 to 2016, were evaluated. The types of anatomical variants of sternal muscle, its origins and insertions, innervation and blood supply were observed. After that, was brought the clinical importance of knowledge of this variation. The sternal muscle has several dimensions and inserts, making difficult to determine its function. Due to its parasternal location in imaging studies it may be seen as an abnormal bulge in the breast region and confused with a tumor. The discovery of this variation before thoracic surgical procedures avoids possible complications, enabling its use as a muscle flap in reconstructive surgery after mastectomy or improving the aesthetic result of breast augmentation providing extra covering for the prosthesis. The knowledge about this anatomical variation is quite relevant, because the perception of the existence of the sternum muscle and its variations is related to important clinical implications in breast and chest surgeries.

Keywords: sternum; anatomic variation; thoracic surgery. 


\section{INTRODUÇÃO}

A presença do músculo esternal, uma variação anatômica, foi primeiramente descrita no século XVII ${ }^{1}$. A incidência desse músculo varia conforme a raça ${ }^{2}$ e o gênero, sendo mais predominante no sexo feminino ${ }^{3}$. Quanto à morfologia, as fibras do músculo esternal geralmente estão dispostas na direção crânio-caudal e o músculo está situado anteriormente ao osso esterno e suas estruturas adjacentes ${ }^{1}$.

Há poucos estudos sobre a origem anatômica, as inserções, as funções e as inervações do músculo esternal ${ }^{4,5}$. Há na literatura mais relatos de estudos anatômicos sobre o músculo esternal do que sobre as implicações clínicas da sua presença ${ }^{6}$; em exames, sua identificação pode ser verificada em mamografia, ultrassom ou durante a palpação para detecção de massas teciduais na parede torácica ${ }^{2}$.

A função do músculo em questão ainda não é definida, mas diversas interpretações são propostas ${ }^{7}$. Assim, o objetivo deste estudo foi fazer uma revisão sistemática da literatura para descrever as variações anatômicas do músculo esternal já identificadas e registradas, apresentando ação, inervação, vascularização e denominações, bem como a importância do conhecimento sobre essas variações na prática clínica.

\section{MÉTODOS}

\section{Estratégia de busca}

Durante as buscas deste estudo foram selecionados e avaliados artigos nos idiomas português e inglês indexados nas bases de dados PubMed (National Library of Medicine e National Institutes of Health) e SciELO (Scientific Electronic Library Online). Foram utilizadas como palavras-chave, nos dois idiomas já mencionados: sternalis muscle, muscular transposition, toracic surgery, anatomic variation, surgical application, mastectomy, breast node, plastic reconstruction, mammography; músculo esternal, transposição muscular, cirurgia torácica, variação anatômica, aplicação cirúrgica, mastectomia, nódulo mamário, reconstrução plástica, mamografia.

\section{Critérios de inclusão}

Durante a busca de informações, foram lidos o título e o resumo dos artigos; para os fins deste estudo, foram eliminadas as duplicidades e os artigos que não se adequaram ao objetivo da presente investigação.

\section{Seleção dos estudos}

Foram revisados artigos originais, revisões da literatura, estudos de casos e ensaios clínicos, com o objetivo de analisar as descrições dos tipos de variações anatômicas do músculo esternal, suas origens e inserções, inervação e irrigação sanguínea. Foram selecionados artigos do período compreendido entre 1867 e 2016 (Quadro 1). A apresentação dos resultados obedeceu ao critério da ordem cronológica da publicação: da mais antiga para a mais recente. Por fim, a discussão dos artigos baseou-se nas variações anatômicas e na importância clínica do músculo esternal.

\section{RESULTADOS}

Após a prospecção dos artigos científicos utilizando os descritores já mencionados para esta revisão, foram encontrados 62 artigos, nos idiomas português e inglês. Após a leitura dos títulos e dos resumos, foi selecionado, conforme critérios de inclusão para esta revisão, o total de 34 artigos.

Devido à variedade de informações encontradas em relação ao músculo esternal, os resultados foram distribuídos no Quadro 1, de acordo com as descrições das variações anatômicas encontradas (ação, inervação, vascularização) ${ }^{8-16}$.

Dessa forma, no Quadro 1 estão dispostos, por ordem de publicação, os artigos que apresentavam estudos de casos in vivo, os quais envolveram exames clínicos e de imagens, além de dissecções cadavéricas.

Cabe salientar que nem sempre todas as informações do Quadro 1 foram trazidas pelos autores, de maneira que há lacunas. Por exemplo, Raiko et al. ${ }^{17}$ relataram que a inervação do músculo esternal é "um pouco enigmática", podendo derivar de diferentes nervos da região torácica, uma vez que depende da localização topográfica da variação anatômica; e Kida et al. ${ }^{11}$ reportaram que, durante a dissecção, é um desafio manter a inervação desse músculo intacta.

\section{DISCUSSÃO}

\section{Variações anatômicas do músculo esternal}

O músculo esternal aparece em cerca de 3 a $8 \%$ da população em geral ${ }^{18}$. Mostra-se mais frequentemente disposto na forma unilateral em 2 a $8 \%$ da população e com menor incidência em sua forma bilateral (1,7\%). Corroborando tal informação, esta revisão encontrou um maior número de menção do músculo na sua variação unilateral, direita ou esquerda ${ }^{9,11-16,18-21}$, quando comparada à forma bilateral ${ }^{5,7-10,12,17-19}$ (Quadro 1).

Em relação às diferentes populações geográficas, sua ocorrência é menor entre os europeus $(4,4 \%)$ e maior entre africanos $(8,4 \%)$ e asiáticos $(11,5 \%)^{21}$. Quanto ao gênero, essa variação atinge mais a população do sexo feminino $(8,7 \%)$ do que do sexo masculino $(6,4 \%)^{3}$.

O músculo esternal é uma variação anatômica bastante estudada por anatomistas, mas ainda pouco familiar para médicos e radiologistas ${ }^{17}$; esse fato ocorre porque anatomistas acabam detectando a variação predominantemente em dissecções cadavéricas ${ }^{4}$. Nessas, há facilidade de identificação desse músculo, já que se trata de um tecido muscular extra no tórax, embora essa visualização possa ser difícil em exames de imagem. A variação em questão, in vivo, quando percebida em exames de imagem, muitas vezes 
é confundida com nodulações ou até mesmo tumores. Isso pode ocorrer devido ao desconhecimento do músculo esternal pelos profissionais especializados para analisar tais exames.

Esse verdadeiro desafio de identificar a presença dessa variação in vivo tem limitado a capacidade de anatomistas para estabelecer sua função ${ }^{18}$, provocando a grande variedade de origens e inserções já descritas na literatura ${ }^{22}$. O músculo em questão representa uma rara variação anatômica ${ }^{23}$ : é um pequeno músculo supranumerário localizado na região torácica anterior ${ }^{15}$, apresenta formato de fita ${ }^{24}$, encontra-se superficial e perpendicularmente ao músculo peitoral maior e paralelo ao osso esterno ${ }^{17}$. Além disso, a classificação do músculo esternal por meio de sua origem durante o período intrauterino ainda é controversa ${ }^{4}$. Assim, diversos autores têm dividido sua origem em quatro categorias principais: a partir do peitoral maior, do reto do abdome, do esternocleidomastoideo e do panículo carnoso ${ }^{10,11}$.

Sobre essa variação anatômica, a literatura apresenta diferentes evidências sobre as dimensões e as inserções do músculo esternal. As origens já relatadas são o esterno, a margem inferior da clavícula, a fáscia do músculo esternocleidomastoideo, o músculo peitoral maior e as costelas superiores e suas cartilagens costais; já as inserções incluem as costelas inferiores e suas cartilagens costais, o músculo peitoral maior, a bainha do músculo reto do abdome e a aponeurose do músculo oblíquo externo do abdome ${ }^{25}$. Patten ${ }^{26}$ traz uma variação no tendão de inserção do músculo esternal, que lança três partes que se inserem no músculo esternocleidomastoideo, e outros que se inserem no corpo das duas clavículas. Desse modo, as diferentes origens e inserções das variações do músculo

Quadro 1: Artigos selecionados conforme critérios de inclusão do presente estudo, trazendo informações sobre a descrição da variação anatômica, ação, inervação e vascularização do músculo estudado

\begin{tabular}{|c|c|c|c|c|}
\hline Autor & Variação anatômica & Ação & Inervação & Vascularização \\
\hline Kirk $^{8}$ & Bilateral & & & \\
\hline Harper $^{9}$ & Unilateral e bilateral & & $\begin{array}{l}\text { Torácico anterior medial } \\
\text { e lateral e terceiro nervo } \\
\text { intercostal }\end{array}$ & \\
\hline Jeng e $\mathrm{Su}^{7}$ & Bilateral & $\begin{array}{l}\text { Auxilia na elevação da parte } \\
\text { inferior da parede torácica }\end{array}$ & $\begin{array}{l}\text { Ramos cutâneos do nervo } \\
\text { intercostal anterior }\end{array}$ & $\begin{array}{l}\text { Ramos da artéria torácica } \\
\text { interna }\end{array}$ \\
\hline $\begin{array}{l}\text { O'Neill e Folan- } \\
\text { Curran }^{10}\end{array}$ & Bilateral & & $\begin{array}{c}\text { Ramos anteriores dos } \\
\text { nervos intercostais }\end{array}$ & \\
\hline Kida et al. ${ }^{11}$ & Unilateral & & $\begin{array}{l}\text { Ramos dos nervos peitoral e } \\
\text { intercostal }\end{array}$ & \\
\hline Jelev et al. ${ }^{12}$ & Unilateral e bilateral & & $\begin{array}{c}\text { Ramos anteriores dos } \\
\text { nervos intercostais }\end{array}$ & $\begin{array}{l}\text { Ramos das artérias } \\
\text { torácicas internas }\end{array}$ \\
\hline Arráez-Aybar et al. ${ }^{13}$ & Unilateral esquerdo & & $\begin{array}{l}\text { Ramos dos nervos cutâneo } \\
\text { anterior e do quarto nervo } \\
\text { intercostal }\end{array}$ & $\begin{array}{l}\text { Ramos dos vasos } \\
\text { intercostais anteriores e da } \\
\text { artéria torácica interna }\end{array}$ \\
\hline Kumar et al. ${ }^{5}$ & Bilateral & & $\begin{array}{l}\text { Ramos do nervo } \\
\text { peitoral medial }\end{array}$ & \\
\hline Kale et al. ${ }^{14}$ & Unilateral & $\begin{array}{l}\text { Lateralização do complexo } \\
\text { mamilo-aréola }\end{array}$ & & \\
\hline Bhat et al. ${ }^{15}$ & Unilateral esquerdo & $\begin{array}{c}\text { Elevar a parte inferior da } \\
\text { caixa torácica }\end{array}$ & & \\
\hline Jetti et al. ${ }^{16}$ & Unilateral direito & & $\begin{array}{l}\text { Terceiro e quarto nervos } \\
\text { intercostais anteriores }\end{array}$ & \\
\hline Raikos et al. ${ }^{17}$ & Bilateral & $\begin{array}{l}\text { Movimento da articulação } \\
\text { do ombro ou acessório na } \\
\text { elevação da parte inferior da } \\
\text { parede torácica }\end{array}$ & & \\
\hline \multirow[b]{2}{*}{ Hung et al. ${ }^{18}$} & Unilateral direito & \multirow{2}{*}{$\begin{array}{l}\text { Elevação da parte inferior } \\
\text { da caixa torácica, agindo } \\
\text { como um acessório da } \\
\text { musculatura inspiratória }\end{array}$} & Nervo peitoral medial & \\
\hline & Bilateral & & $\begin{array}{c}\text { Ramos anteriores } \\
\text { dos segundos nervos } \\
\text { intercostais }\end{array}$ & \\
\hline Liu et $a l .{ }^{19}$ & Bilateral & $\begin{array}{l}\text { Músculo acessório na } \\
\text { elevação da caixa torácica }\end{array}$ & $\begin{array}{c}\text { Ramos dos quarto, quinto } \\
\text { e sexto nervos intercostais } \\
\text { anteriores }\end{array}$ & \\
\hline Nguyen e Ogawa ${ }^{20}$ & Unilateral esquerdo & & $\begin{array}{c}\text { Ramos do nervo torácico e/ } \\
\text { ou nervo intercostal }\end{array}$ & Artéria torácica anterior \\
\hline Silveira et al. ${ }^{21}$ & Unilateral esquerdo & $\begin{array}{l}\text { Corrugar a pele da parte } \\
\text { anterior da parede torácica }\end{array}$ & & \\
\hline Anjamrooz ${ }^{24}$ & Unilateral & $\begin{array}{l}\text { Auxilia na elevação das } \\
\text { costelas inferiores }\end{array}$ & $\begin{array}{c}\text { Ramos torácicos anteriores } \\
\text { dos nervos intercostais } \\
\text { direitos }\end{array}$ & \\
\hline Katara et al. ${ }^{23}$ & Unilateral direito & $\begin{array}{c}\text { Elevação da parte inferior da } \\
\text { parede torácica }\end{array}$ & Nervo peitoral & \\
\hline
\end{tabular}


esternal já descritas na literatura, com suas origens embrionárias, tendem a dificultar um consenso acerca da função desse músculo.

No geral, quando um músculo é contraído, a origem corresponde ao ponto fixo, e a inserção, ao ponto móvel. Portanto, supõe-se que a contração do músculo esternal possa exercer um papel acessório na elevação da margem inferior da caixa torácica ${ }^{23,27,28}$, atuando consequentemente como um músculo acessório da inspiração ${ }^{19}$. Outra possibilidade é de que essa estrutura reforce a função do peitoral maior ${ }^{28}$.

Esta revisão observou divergência na literatura quanto às funções do músculo esternal (Quadro 1). A maioria dos autores aponta que o músculo auxilia na elevação da parte inferior da parede torácica, elevando, assim, as costelas inferiores ${ }^{7,15,17-22,23}$, agindo como musculatura acessória da respiração ${ }^{18}$; porém há menção de que essa variação possa corrugar a pele da parte anterior da parede torácica ${ }^{21} \mathrm{ou}$, ainda, participe de movimentos da articulação do ombro ${ }^{17}$. Em adição a isso, Kale et al..$^{14}$ mencionam uma variação capaz de realizar a lateralização do complexo mamilo-aréola. Contribuem para essa divergência de funções do músculo esternal: a dificuldade de identificá-lo em exames que utilizam imagens; a ausência de um exame físico ou clínico que possibilite uma identificação de função para o músculo em questão; e, por fim, a presença de inúmeras variações para um mesmo músculo.

Além do obstáculo no consenso para a funcionalidade do músculo esternal, há discordância quanto à sua denominação desde as primeiras descrições na literatura, feitas por Cabrolius, em $1604^{27}$, e por Du Puy, em $1726^{15}$. Diferentes denominações têm sido empregadas ao músculo esternal, referindo-se a sua localização, origem e inserção ou morfologia: musculus sternalis, episternalis, parasternalis, presternalis, rectus sternalis, rectus sterni, rectus thoracis, rectus thoracicus superficialis, superficial rectus abdominis, sternalis brutorum, japônicas, thoracicus ${ }^{25}$, accesorius ad rectum, anomalus sterni, episternal, pectoris rectus, supracostalis, triangular muscle anterior e external to sternum ${ }^{13}$. Ainda, Kale et al. ${ }^{14}$ relataram a existência de um outro tipo de variação anatômica do músculo esternal. Durante uma cirurgia de redução de mama, esses autores nomearam o músculo encontrado como sternomastalis. Uma estrutura músculo-tendínea foi descoberta no interior da mama direita, com origem a partir da fáscia peitoral e da junção esternocostal e inserção no complexo mamilo-aréola.

Igualmente às divergências nas funções e denominações para o músculo esternal, há suposições, por meio de dissecações cadavéricas e explorações cirúrgicas, de que a inervação é proveniente dos nervos peitorais ou dos ramos anteriores dos nervos intercostais, podendo também ser a combinação de ambos ${ }^{18}$; porém a maioria dos músculos esternais é inervada por ramos do nervo peitoral, ainda que a contribuição de ramos do nervo intercostal seja frequente ${ }^{11}$. Já o suprimento sanguíneo desse músculo é originário dos ramos da artéria torácica interna ${ }^{7,12,13,20}$.

Dessa maneira, Jelev et al. ${ }^{12}$ sugerem que, para ser aceito como um músculo esternal, devem ser apresentadas as seguintes características, independentemente do comprimento e da espessura:

1. localizar-se entre a fáscia superficial da região torácica anterior e da fáscia peitoral;
2. ter origem no esterno ou na região infraclavicular;

3. inserir-se nas costelas inferiores, nas cartilagens costais, na aponeurose do músculo oblíquo externo do abdome ou na bainha do músculo reto do abdome;

4. apresentar inervação proveniente dos nervos torácico anterior (peitoral) e/ou intercostais.

Para esta revisão, foi observado, nos artigos encontrados e dispostos no Quadro 1, que todos os músculos esternais estudados pelos autores ${ }^{5,7-23}$ se encaixam em pelo menos um dos critérios propostos por Jelev et al. ${ }^{12}$, podendo, portanto, ser considerados como variações anatômicas do músculo esternal. Sendo assim, o músculo esternal não é apenas uma rara variação muscular com ação pouco definida, mas também uma estrutura com grande importância clínica ${ }^{12}$. Todavia, é um músculo relativamente desconhecido por clínicos, uma vez que a literatura aponta que a discussão sobre o músculo em questão é quase inexistente durante a formação médica ${ }^{13}$.

\section{Importância clínica do músculo esternal}

$\mathrm{O}$ desafio de identificar a presença do músculo esternal in vivo tem limitado a capacidade dos anatomistas para estabelecer sua função ${ }^{18}$. Mesmo com a observação relatada por $\mathrm{Kirk}^{8}$, na qual o músculo esternal aparece contraído em uma foto de um indivíduo realizando a flexão do tronco associada à abdução dos braços, os demais casos encontrados em viventes e publicados ainda são, em sua maioria, achados acidentais durante operações cirúrgicas ou exames de imagem.

A tomografia computadorizada é um importante exame capaz de detectar a presença do músculo esternal. Um estudo realizado em 2014 verificou que esse músculo foi altamente prevalente em adultos chineses. Por meio de tomografias computadorizadas, os autores avaliaram a presença do músculo esternal em 6.000 chineses, e verificaram que ele estava presente em 347 (5,8\%) dos indivíduos pesquisados, sendo bilateral em 118 (34,0\%), unilateral direito em 148 (42,7\%) e unilateral esquerdo em 81 (23,3\%). Também foi possível avaliar a morfologia, a origem e a inserção desse músculo 29 .

A compreensão dessa variação anatômica é importante para os terapeutas, pois, quando o músculo esternal origina-se do músculo esternocleidomastoideo, supõe-se que haja a inspiração acessória automática com a rotação da cabeça ${ }^{19}$. Assim, o músculo classificado como bíceps esternal, encontrado por Anjamrooz ${ }^{24}$, realizaria a elevação das costelas inferiores, o que também poderia contribuir como músculo acessório na inspiração. O músculo esternal ainda é relatado como capaz de alterar um exame de eletrocardiograma (ECG) ${ }^{25}$.

Contudo, as maiores implicações do músculo esternal para a clínica são verificadas em cirurgias de mama e tórax. Devido à localização paraesternal, em exames de imagem, o músculo pode ser visto como uma nodulação anormal na região da mama e ser confundido com um tumor ${ }^{1,30}$. Para facilitar ou até mesmo permitir a 
diferenciação do músculo esternal de uma real doença, como um possível tumor, Bradley et al. ${ }^{31}$ e Kumar et al. ${ }^{5}$ sugerem o uso concomitante de ressonância magnética, mamografia ou tomografia. Ainda, dependendo da localização, pode haver invasão de tumores mamários nesse músculo, tornando necessária a sua remoção cirúrgica durante mastectomias ${ }^{4}$.

Os cirurgiões mastologistas estão pouco habituados com a presença desse músculo extranumerário. Ressalta-se que o conhecimento sobre o músculo esternal pelo profissional é muito importante no ato cirúrgico, pois auxilia a identificação precoce dos planos anatômicos durante uma cirurgia mamária e possibilita um plano de dissecção apropriado ${ }^{1,23}$; quando não detectado, pode interferir em procedimentos, resultando em períodos operatórios mais longos ${ }^{25}$.

Schulman e Chun ${ }^{6}$ relataram que, apesar de ser uma variação anatômica rara, durante 15 anos de carreira como cirurgiões encontraram cerca de 6 vezes o músculo esternal em cirurgias de reconstrução de mamas. No entanto, Bailey e Tzarnas ${ }^{30}$, em pesquisa realizada com 65 médicos e estudantes de Medicina, constataram uma falta geral de familiaridade com o músculo esternal.

A importância de conhecer a existência da variação anatômica em questão pode ser observada no trabalho de Harish e Gopinath ${ }^{31}$, em que os autores investigaram registros do pós-operatório de 1.152 pacientes que fizeram mastectomia no período de 1990 até 2000 e encontraram registros de oito mulheres que possuíam distintas variações do músculo esternal. Por meio de exames utilizando imagens e consequente conhecimento prévio da existência da variação pelos cirurgiões, todos os músculos, apesar de possuírem morfologia diferente entre as pacientes, puderam ser bem preservados após a mastectomia.
Ainda, quando detectado no pré-operatório, o músculo esternal pode ser usado como um retalho muscular na cirurgia reconstrutiva após a mastectomia e melhorar os resultados estéticos da mamoplastia de aumento, fornecendo cobertura extra para a prótese e proporcionando uma aparência mais natural para a mama ${ }^{4,25}$. Por outro lado, no caso de um cirurgião pouco experiente, a presença do músculo esternal pode interferir na dissecção, aumentando o tempo de cirurgia ${ }^{4}$.

O conhecimento sobre o aspecto do músculo esternal em exames de imagens, como ultrassom e mamografias, pode permitir o diagnóstico precoce e impedir uma avaliação mais aprofundada desnecessária, como, por exemplo, uma biópsia²; além de permitir ao cirurgião que se deparar com essa variação anatômica a possibilidade de adaptar sua técnica cirúrgica à presença desse músculo acessório ${ }^{6}$.

Portanto, a familiaridade com o seu aspecto é essencial para evitar confusão com uma ampla variedade de lesões, tais como carcinoma da mama, hematomas, linfadenite, necrose de gordura e cicatrizes cirúrgicas ${ }^{22}$. Sendo assim, o conhecimento acerca desse músculo é de grande relevância para mastologistas, cirurgiões plásticos e radiologistas especializados em imagem da mama, pois ajudará no diagnóstico, evitando erros médicos, bem como poderá auxiliar diretamente no processo cirúrgico ${ }^{1,15,18,32,33}$.

Dessa forma, conclui-se que o conhecimento sobre essa variação anatômica é bastante relevante para diversos profissionais da área da saúde, devido às grandes implicações clínicas nas cirurgias de mama e tórax. Para um melhor cuidado interprofissional, é necessário que profissionais de saúde de diversas especialidades tenham conhecimento sobre essa variação anatômica.

\section{REFERÊNCIAS}

1. Marques EF, Souza JA, Graziano L, Bitencourt AGV, Senaga C, Fontes CEM. Sternalis muscle simulating a breast nodule. Rev Bras Ginecol Obstet. 2009;31(10):492-5. http://dx.doi.org/10.1590/S0100-72032009001000004

2. Nuthakki S, Gross M, Fessell D. Sonography and helical computed tomography of the sternalis muscle. J Ultrasound Med. 2007;26(2):247-50

3. Scott-Conner CE, Al-Jurf AS. The sternalis muscle. Clin Anat. 2002;15(1):67-9.

http://dx.doi.org/10.1002/ca.1096

4. Salval A, Scevola A, Baruffaldi Preis FW. Sternalis muscle: an uncommon finding during aesthetic breast surgery. Aesthet Surg J. 2012;32(7):903-5. http://dx.doi.org/10.1177/1090820X12455048

5. Kumar H, Rath G, Sharma M, Kohli M, Rani B. Bilateral sternalis with unusual left-sided presentation: a clinical perspective. Yonsei Med J. 2003;44(4):719-22. http://dx.doi.org/10.3349/ymj.2003.44.4.719

6. Schulman MR, Chun JK. The conjoined sternalis-pectoralis muscle flap in immediate tissue expander reconstruction after mastectomy. Ann Plast Surg. 2005;55(6):672-5.
7. Jeng $\mathrm{H}$, Su SJ. The sternalis muscle: an uncommon anatomical variant among Taiwanese. J Anat. 1998;193(Pt 2):287-8. http://dx.doi.org/10.1046/j.1469-7580.1998.19320287.x

8. Kirk TS. Sternalis Muscle (in the Living). J Anat. 1925;59(Pt 2):192.

9. Harper WF. The Sternalis Muscle in the Anencephalous Foetus. J Anat. 1936;70(Pt 2):317-20.

10. O'Neill MN, Folan-Curran J. Case report: bilateral sternalis muscles with a bilateral pectoralis major anomaly. J Anat. 1998;193(Pt 2):289-92.

http://dx.doi.org/10.1046/j.1469-7580.1998.19320289.x

11. Kida MY, Izumi A, Tanaka S. Sternalis muscle: topic for debate. Clin Anat. 2000;13(2):138-40.

http://dx.doi.org/10.1002/(SICI)1098-2353(2000)13:2<138::AIDCA8 $>3.0 . C O ; 2-4$

12. Jelev L, Georgiev G, Surchev L. The sternalis muscle in the Bulgarian population: classification of sternales. J Anat. 2001;199(Pt 3):359-63. http://dx.doi.org/10.1046/j.1469-7580.2001.19930359.x

13. Arráez-Aybar LA, Sobrado-Perez J, Merida-Velasco JR. Left musculus sternalis. Clin Anat. 2003;16(4):350-4. http://dx.doi. org/10.1002/ca.10120 
14. Kale SS, Herrmann G, Kalimuthu R. Sternomastalis: a variant of the sternalis. Ann Plast Surg. 2006;56(3):340-1. http://dx.doi.org/10.1097/01.sap.0000197567.42535.d9

15. Bhat KM, Potu BK, Gowda S. Sternalis muscle revisted in South Indian male cadaver: a case report. Cases J. 2009;2:6318. http://dx.doi.org/10.4076/1757-1626-2-6318

16. Jetti R, Pamidi N, Vollala VR, Vasavi R, Nerella VM. Right sternalis muscle. Int J Anat Variations. 2009;2:41-2.

17. Raikos A, Paraskevas GK, Tzika M, Faustmann P, Triaridis S, Kordali $\mathrm{P}$, et al. Sternalis muscle: an underestimated anterior chest wall anatomical variant. J Cardiothorac Surg. 2011;6:73. http://dx.doi.org/10.1186/1749-8090-6-73

18. Hung LY, Lucaciu OC, Wong JJ. Back to the debate: sternalis muscle: volver al debate: músculo esternal. Int J Morphol. 2012;30(1):330-6

19. Liu HH, Holmes $\vee$, Nordon-Craft A, Reeves R. Variation of sternalis muscle: a case report. Intern J Anat Variation. 2012:5:59-61.

20. Nguyen DT, Ogawa R. The sternalis muscle-incidental finding of a rare chest wall muscle variant during keloid excision-chest wall reconstruction. Eplasty. 2012;12:e36.

21. Silveira D, Sousa LM, Siqueira SL, Oliveira BVM, Silva AT, Costa JP, et al. Sternalis muscle: an anatomic variation of the anterior chest wall. J Morphol Sci. 2012;29(2):76-8

22. Silva RG. Variação anatômica do músculo esternal: anatomia clínica e revisão da literatura. Monografia (Conclusão de Curso) Faculdade de Medicina da Bahia da Universidade Federal da Bahia, Salvador, 2013

23. Katara $P$, Chauhan S, Arora R, Saini P. A unilateral rectus sternalis muscle: rare but normal anatomical variant of anterior chest wal musculature. J Clin Diagn Res. 2013;7(12):2665-7. http://dx doi org/10.7860/JCDR/2013/7379.3726
24. Anjamrooz SH. Biceps sternalis: a Y-shaped muscle on the anterior chest wall. J Cardiothorac Surg. 2013;8:38. http://dx.doi.org/10.1186/1749-8090-8-38

25. Snosek M, Tubbs RS, Loukas M. Sternalis muscle, what every anatomist and clinician should know. Clin Anat. 2014;27(6):866-84 http://dx.doi.org/10.1002/ca.22361

26. Patten CJ. Right sternalis muscle with expanded fenestrated tendon. J Anat. 1934;68(Pt 3):424-5.

27. Turner WM. On the musculus sternalis. J Anat Physiol. $1867 ; 1(2):[246]-253$

28. Raikos A, Paraskevas GK, Yusuf F, Kordali P, loannidis O, BrandSaberi B. Sternalis muscle: a new crossed subtype, classification, and surgical applications. Ann Plast Surg. 2011;67(6):646-8. http://dx.doi.org/10.1097/SAP.0b013e31820d688b

29. Ge Z, Tong Y, Zhu S, Fang X, Zhuo L, Gong X. Prevalence and variance of the sternalis muscle: a study in the Chinese population using multi-detector CT. Surg Radiol Anat. 2014;36(3):219-24. http://dx.doi.org/10.1007/s00276-013-1175-4

30. BaileyPM, Tzarnas CD. The sternalismuscle: anormal finding encountered during breast surgery. Plast Reconstr Surg. 1999;103(4):1189-90.

31. Harish K, Gopinath KS. Sternalis muscle: importance in surgery of the breast. Surg Radiol Anat. 2003;25(3-4):311-4. http://dx.doi.org/10.1007/s00276-003-0119-9

32. Shiotani M, Higuchi T, Yoshimura N, Kiguchi T, Takahashi N, Maeda $\mathrm{H}$, et al. The sternalis muscle: radiologic findings on MDCT. Jpn J Radiol. 2012;30(9):729-34. http://dx.doi.org/10.1007/s11604-012-0114-3

33. Bradley FM, Hoover Jr. HC, Hulka CA, Whitman GJ, McCarthy KA, Hall DA, et al. The sternalis muscle: an unusual normal finding seen on mammography. AJR Am J Roentgenol. 1996;166(1):33-6. http://dx.doi.org/10.2214/ajr.166.1.8571900 